\title{
La evaluación de la comunicación científica escrita desde una perspectiva didáctica en la enseñanza posgraduada*
}

Assessment of written scientific communication from a teaching perspective in postgraduate education

María del Carmen Navarrete Reyes ${ }^{\star \star}$

Juana Idania Pérez Morales***

Dalila González Rodríguez****

\section{Resumen:}

El proyecto desarrolla un entrenamiento para la producción de textos científicos argumentativos.

El proyecto «Estrategia didáctica para potenciar en los profesionales las habilidades comunicativas para la comunicación científica», desarrolla un entrenamiento para la producción de textos científicos argumentativos. En el artículo se exponen los indicadores de evaluación que emplearán el profesor y los estudiantes para evaluar el proceso de producción del texto científico argumentativo y su calidad.

*Profesora Titular. Profesora de Lengua Rusa y Español como lengua extranjera. Coordinadora de la línea de investigación «Competencia comunicativa e intercultural en el estudio de lenguas» y el proyecto «Estrategia didáctica para potenciar en los profesionales las habilidades comunicativas para la comunicación científica», Facultad de Humanidades, Universidad Central «Marta Abreu » de Las Villas, Cuba. mcnavarr@uclv.edu.co

**Profesora Titular. Profesora de Lengua y Literatura Rusa e Inglesa. Dirige el proyecto «Lengua y competencia comunicativa», grupo de Evaluación del aprendizaje, Facultad de Humanidades, Universidad Central «Marta Abreu » de Las Villas. juanap@uclv.edu.cu

***Asistente . Filóloga en Lengua Hispanoamericana. Miembro del proyecto «Estrategia didáctica. Profesora ctica para potenciar en los profesionales las habilidades comunicativas comunicativas dalila@uclv.edu.cu 


\section{Palabras clave: Comunicación científica escrita, evaluación, indicadores de evaluación, texto científico escrito.}

\section{Abstract}

The project «Teaching strategy to enhance scientific communication skills in professionals» develops a training course for the production of argumentative scientific texts. The paper presents the assessment criteria to be applied by the teacher and students for assessing the writing production process and the quality of argumentative scientific texts.

\section{Keywords: Scientific writing communication, assessment, assessment criteria, scientific writing text.}

\section{Introducción}

La evaluación es el componente que en gran medida determina el qué y el cómo el alumno aprende. Constituye una herramienta que orienta el aprendizaje del estudiante y la reflexión compartida del docente con los alumnos para alcanzar los objetivos que se planteen. La participación en calidad de tutoras, oponentes y miembros de tribunales en defensas de tesis, ha permitido evidenciar que los tesiantes no siempre tienen desarrolladas las habilidades comunicativas, lo cual influye desfavorablemente en el nivel de comprensión del mensaje que se transmite.

Ante esta problemática se elaboró el proyecto «Estrategia didáctica para potenciar en los profesionales las habilidades comunicativas para la comunicación científica», cuyo objetivo es potenciar las habilidades comunicativas en los profesionales e investigadores para que comuniquen con claridad, precisión, coherencia y objetividad los resultados del trabajo investigativo a la comunidad científica.

Uno de los resultados planificados en este proyecto es un entrenamiento para la producción de textos científicos argumentativos, donde la evaluación como com- ponente del proceso de enseñanza-aprendizaje desempeñe un papel fundamental. Se propone que esta se realice sistemáticamente durante todo el desarrollo del entrenamiento; que el profesor y los alumnos sean sujetos activos y reflexionen de manera conjunta; que los alumnos autoevalúen los textos científicos producidos por ellos con la ayuda de indicadores que les propicie el profesor, los cuales deben estar en correspondencia con la instrumentación de la tarea previamente concebida por el docente. Los indicadores serán comunes para el profesor y los alumnos. Así, la evaluación que realiza el docente será más objetiva si se corresponde con la autoevaluación y coevaluación que hizo el alumno de su actividad.

En el presente trabajo se expone, como un avance de la investigación, una propuesta de indicadores de evaluación que permitan al profesor y a los estudiantes valorar el proceso de producción del texto científico escrito y la calidad del mismo.

\section{Fundamentos teóricos}

\subsection{Conceptualización de la evaluación}

Según Coll, C. \& Onrubia, J. (1999) la evaluación es un proceso sistemático de análisis y valoración de los progresivos cambios que ocurren en los alumnos por la acción educativa y, además, constituye una vía de obtención de información para tomar decisiones orientadas al mejoramiento de la actividad educativa. González, M. (2000) refiere que la evaluación es una actividad humana y que como cualquier actividad, su modo de existencia es dinámico, como proceso. Añade que es el proceso y resultado de juzgar la valía de un objeto o fenómeno de la realidad en sus características esenciales, sus manifestaciones particulares, su devenir, estado de desarrollo actual y previsible, de 
acuerdo con criterios de referencia pertinentes a la naturaleza del propio objeto y a los propósitos que se persigan.

Por su parte, Sanmartí, J. (1996, citado en Pérez Cabaní M. y Álvarez 1.:2000) considera la evaluación como un proceso que se desarrolla en tres etapas e implica: recogida de información a partir de criterios preestablecidos, análisis de esta información y juicio sobre el resultado de este análisis y toma de decisiones de acuerdo con el juicio emitido.

En las definiciones expuestas resulta coincidente la visión que se da de la evaluación como proceso, criterio que es compartido por las autoras de este trabajo, para quienes la evaluación es el componente del proceso de enseñanza-aprendizaje que está presente en todas sus etapas: planificación, organización, ejecución y evaluación; tiene la función de retroalimentar sistemáticamente a los agentes de dicho proceso -alumnos, profesores, autoridades educativas- sobre la calidad de este con la finalidad de que se tomen las decisiones para mejorarlo.

En las definiciones expuestas de González, M. (2000) y Sanmartí, J. (1996) se pone de manifiesto la importancia que se le concede a los criterios de evaluación, ya que sirven de base tanto para la recogida, análisis y juicio valorativo de la información, como para la comunicación de los resultados y la toma de decisiones. Los criterios de evaluación constituyen la base para establecer los indicadores de evaluación.

\subsection{Evaluación y actividad científico-investigativa}

La evaluación como indicador de la calidad de los sistemas educativos está presente en la actividad científico-investigativa que desarrollan los estudiantes (en programas de especialidad, maestría, doctorado). Esta debe recoger evidencias sobre el dominio que el estudiante tiene de las habilidades investigativas y comunicativas, las cuales se requieren para la determinación, planteamiento y formulación del problema, objetivo, objeto de investigación e hipótesis que defiende; selección y determinación de los métodos, instrumentos y muestras de investigación. Además, para la fundamentación teórica del trabajo mediante análisis crítico y toma de posición; estructuración del trabajo en correspondencia con el diseño; elaboración del informe escrito y presentación oral de los resultados.

La evaluación debe favorecer la adecuada interrelación de las habilidades mencionadas anteriormente para garantizar el éxito del trabajo científico-investigativo, ya que las mismas se manifiestan y desarrollan en forma de sistema.

Las habilidades investigativas y comunicativas conforman las competencias investigativa y comunicativa, imprescindibles para el desarrollo de la labor científico-investigativa que desarrolla el estudiante en su formación profesional.

De lo expuesto se evidencia la necesidad de que el estudiante sea competente en la lengua materna y en particular, domine el lenguaje científico, para lo cual requiere conocimientos, habilidades, capacidades y convicciones relacionadas con el discurso científico, aspectos que deben ocupar un lugar destacado en las propuestas curriculares de los diferentes niveles de enseñanza y programas de formación académica.

\section{Evaluación y discurso científico}

Evaluar el discurso científico resulta un objeto complejo. Según Cassany, D. el discurso científico es «un tipo de discurso altamente elaborado, caracterizado por el uso del registro formal de la lengua, por utilizar un lenguaje objetivo con un léxico preciso y específico» (1999).

El discurso científico se fundamenta en argumentos sólidos y rigurosos y para ello emplea el lenguaje científico: «conjunto de registros especializados, propios de las diversas ciencias, caracterizados por unas opciones específicas en el léxico, la sintaxis y la organización discursiva que los diferencian de la lengua común. Un factor diferencial del lenguaje científico es la realidad de la que se habla» (Roméu, A.:2002). Predomina el carácter abstracto-generalizador, la objetividad, la rigurosidad, la lógica de la exposición, 
la enseñanza posgraduada

la coherencia y cohesión, la precisión semántica, la emocionalidad oculta, el escaso uso de sinónimos, el empleo de términos, nomenclatura, símbolos, el predominio de la oración simple, la escasa utilización de la subordinación y la impersonalidad.

La práctica sistemática de la evaluación del aprendizaje con fines formativos puede contribuir a una mejor construcción del discurso científico. Por ello, en la evaluación de la comprensión y construcción del discurso científico se pueden utilizar procedimientos, tales como: expresar el sentido global del texto, identificar las ideas generales, relacionar las ideas, parafrasear el texto, explicar hechos, interpretar unidades textuales y el texto globalmente, comparar, argumentar posiciones, extrapolar lo leído a situaciones reales, criticar y tomar posición, dialogar con los autores, valorar y construir el discurso propio.

\section{Evaluación de la comunicación científica escrita}

La evaluación como proceso sistemático y regulador permite recoger información sobre la comunicación científica escrita, valorarla y tomar las decisiones para mejorar el proceso de producción del texto científico y la calidad, así como la presentación y comunicación escrita de los resultados a la comunidad científica.

Con este propósito la evaluación debe valorar, entre otros aspectos, si en el escrito se expresan criterios personales argumentados y razonados, si se reconocen las aportaciones de los autores; si la producción científica escrita está documentada y cómo en ella se expresa la utilización de las fuentes de información científica, el análisis y uso de la información; cómo se describen los resultados originales de la investigación, y si estos pueden ser comprendidos y utilizados por otros investigadores.

Además debe tener en cuenta los géneros y tipos de textos que los estudiantes emplean para comunicar lo anteriormente expuesto, así como los conocimientos, habilidades, capacidades, convicciones y procedimientos que se requieren para la comprensión y producción del texto científico.
La labor educativa muestra que, «a pesar de que en las últimas décadas ha sido posible constatar un notable perfeccionamiento de las prácticas evaluativas [...]» (Gimeno, 1999, citado en Tapia, M. y col. ,2003), la evaluación de la comunicación científica escrita por lo general se reduce a la valoración, fundamentalmente holística, del texto científico y de los aspectos ortográficos, lexicales, morfológicos y sintácticos, lo cual no revela las insuficiencias y dificultades que tienen los estudiantes en este tipo de comunicación.

Ante esta situación se considera que la utilización de indicadores de evaluación es útil, pues estos pueden orientar y contribuir a que la evaluación y autoevaluación que realizan los profesores y los estudiantes sea más objetiva y evidencie las debilidades y fortalezas de la producción y calidad de la comunicación científica escrita.

La propuesta de indicadores de evaluación que a continuación se presenta parte del estudio de las características y rasgos lingüísticos del texto científico, y en particular del tipo argumentativo. Además, se toman como patrones los planteados por Albarrán (2008), los cuales fueron adaptados a los objetivos del entrenamiento. Indicadores para evaluar el proceso de producción de textos científicos escritos. Se relacionan con las operaciones cognitivas que tienen lugar en las etapas del proceso de escritura: planificación, textualización y revisión.

Etapa de planificación. Es una operación mental que realiza el profesional antes, durante y después de la textualización o revisión. Incluye la generación y organización de las ideas y criterios descubiertos sobre el tema investigado, de los planteamientos, de las informaciones y datos recogidos; la formulación de los objetivos que se pretenden lograr con el texto científico escrito y la determinación de los receptores de la comunicación escrita. 
Indicadores de evaluación de la planificación:

- genera ideas con la ayuda de técnicas: lluvia de ideas, mapa de ideas, escritura libre, dibujos;

- organiza las ideas a través de técnicas: organizadores gráficos (mapas conceptuales, diagrama de Venn, tablas y esquemas), categorización de las ideas, numeración de las ideas (flechas, corchetes, viñetas);

- plantea en el borrador de manera clara y precisa los objetivos que se pretenden lograr con el texto escrito;

-revela la estructura lógica del texto mediante subtemas, subtítulos;

- propone un título tentativo para el texto escrito.

\section{Etapa de textualización}

Es la operación mental mediante la cual se desarrollan diversos procesos cognitivos para que las ideas, criterios, opiniones, planteamientos, conocimientos, informaciones, datos, queden registrados en un papel o digitalmente.

\section{Indicadores de evaluación de la textualización:}

- ejecuta el plan elaborado

- incluye las citas de la revisión de la literatura;

- usa una norma para la referencia bibliográfica;

- incluye gráficos, ilustraciones;

- elabora el resumen;

- realiza las versiones necesarias.

Etapa de la revisión. Es la actividad mental que realiza el escritor a lo largo de las etapas de planificación del texto y textualización.

Indicadores de evaluación de la revisión durante la etapa de planificación

-agrega nuevas ideas, criterios, opiniones, planteamientos, datos, a las generados;

-elimina ideas, criterios, opiniones, planteamientos y datos generados;

-compara las nuevas ideas, criterios, opiniones, planteamientos y datos con los generados;

-realiza correcciones en las ideas, criterios, opiniones, planteamientos y datos organizados;
- reformula y reescribe las ideas, criterios, opiniones, planteamientos y datos.

Indicadores de evaluación de la revisión durante la etapa de textualización

- elimina ideas argumentadas o ejemplificadas;

- agrega nuevos argumentos o ejemplos a las ideas expuestas;

- amplía las argumentaciones o ejemplificaciones a las ideas expuestas;

- excluye las ideas repetidas;

- señala las ideas contradictorias;

- confirma que existe una idea principal en cada párrafo;

- verifica la existencia de una introducción, desarrollo y conclusión;

- confirma que existe relación entre el título y el contenido, entre los objetivos y el contenido, entre los objetivos y las conclusiones, entre las conclusiones y las recomendaciones, entre el título, el problema, los objetivos, las conclusiones y las recomendaciones; entre el problema y el objetivo;

-constata que los conectores están utilizados adecuadamente y son variados;

- confirma que utiliza los pronombres de forma adecuada;

- comprueba que mantiene a lo largo del texto la condición de tiempo verbal;

- constata que el registro es el formal y está acorde al contexto donde será comunicado;

- muestra una revisión del uso correcto de los signos de puntuación:

- emplea el léxico de la ciencia a la cual corresponde el tema así como la terminología y la nomenclatura, define los conceptos;

- corrige la morfosintaxis;

- corrige la ortografía.

Indicadores para evaluar la calidad del producto. Se relacionan con el producto de la actividad mental: la textualización. Se relacionan con el contenido, las habilidades cognitivas y cognitivo-lingüísticas, coherencia y cohesión, adecuación, género textual, tipología textual, léxico, morfosintáxis, ortografía y edición del texto. 
Contenido. Es el fondo del texto escrito: las ideas, las opiniones, la información, los datos, entre otros, que se hacen visibles en el texto producido, Para hacerlo el profesional necesita poner en práctica las habilidades cognitivas y cognitivo-lingüísticas.

Indicadores de evaluación del contenido y las habilidades cognitivas y cognitivo-lingüísticas

-aporta suficientes ideas relacionadas con el tema;

-argumenta las ideas principales;

- manifiesta desarrollo de las habilidades cognitivas (analizar, comparar, clasificar, identificar, interpretar, inferir, deducir, transferir, valorar, operar) empleadas en el texto escrito;

- manifiesta desarrollo de las habilidades cognitivolingüísticas (describir, definir, resumir, explicar, justificar, argumentar y demostrar) utilizadas en el texto escrito.

Coherencia. Tiene un carácter pragmático y semántico. Significa mantener unidas las ideas que se presentan en el texto; le permite al lector pasar fácilmente de una oración a otra, de un párrafo a otro sin sentir que existan lagunas en el pensamiento, ni saltos bruscos. La coherencia representa la eliminación de toda contradicción en un conjunto de ideas.

Indicadores de evaluación de la coherencia

\section{Información:}

- evita los enunciados contradictorios;

-menciona en el texto correctamente los cuadros, gráficos, esquemas;

-evita frases ambiguas;

-adecua el título al contenido;

\section{Estructura:}

- construye el texto con una estructura de acuerdo a la tipología textual;

- evita ideas repetidas;

- ordena los hechos o las ideas de forma lógica;

\section{Párrafos:}

- mantiene el tema a lo largo del texto (progresión temática);

- presenta una idea principal en cada párrafo;

-escribe párrafos de no más de tres o cuatro oraciones;

\section{Cohesión:}

Es la relación que existe entre los elementos que forman parte del texto (palabras, oraciones y párrafos) que contribuyen a la interpretación del sentido del texto por parte del receptor.

Indicadores de evaluación de la cohesión

-utiliza correctamente los signos de puntuación;

-emplea mecanismos de cohesión adecuadamente:

- preposiciones (enumeración, secuencia, reformulación, adición, comparación, sustitución de pronombres, causa, consecuencia y preposiciones);

- conjunciones;

- palabras generalizadoras;

- mantiene a lo largo de la texto escrito la condición del tiempo verbal (pasado, presente y futuro);

- tiene presente la condición del pronombre a lo largo del texto escrito.

Adecuación. Es la adaptación del registro al contexto donde se encuentra el receptor. Tiene un carácter pragmático y semántico al igual que la coherencia. Indicadores de evaluación de la adecuación

-hay correspondencia con la estructura del género textual: artículo científico, monografía científica, tesis doctoral, tesis de maestría;

-hay correspondencia con el registro del género textual;

- hay correspondencia con la temática de la especialidad;

- se emplea la terminología de la especialidad correspondiente;

- es impersonal;

- se emplean adecuadamente iconos, códigos numéricos, código verbal: negrito, cursiva, entrecomillado. 
Género textual. Alude a la división de la literatura en general, o al patrón que se sigue para componer el texto escrito.

Indicador de evaluación del género textual

- elije el género textual adecuado al fin que se quiere lograr y a la tipología textual.

Se ejemplifica con el artículo científico por ser el género textual más prestigioso en el ámbito científico internacional, y el medio más importante con que cuenta el investigador para comunicar a la comunidad científica los resultados de las investigaciones. Introducción:

Título

- se escribe de forma clara y precisa;

- permite identificar el tema;

-es corto.

Autores

- aparecen los autores del artículo;

- aparece la dirección de contacto de los autores.

Resumen

- aparece el resumen y las palabras clave (de 3 a 10);

- o orienta el contenido fundamental del artículo;

- o se expresan de manera clara y breve los objetivos, el alcance del estudio, los procedimientos básicos, los métodos empleados, los principales hallazgos y las conclusiones;

- se emplea la tercera persona, forma impersonal, tiempo pasado;

- se excluyen las abreviaturas y las referencias.

Abstract

- aparece el abstract con el mismo texto del resumen y las palabras clave en idioma inglés (de 3 a 10).

Introducción

- se identifica el problema o cuestión que trata el artículo;

- se exponen los trabajos más relevantes sobre el tema que se trata;

- se exponen las contribuciones de otros autores al tema que se trata;

- se justifica el estudio del tema;

- se formulan las hipótesis y los objetivos;

- se emplea la tercera persona, forma impersonal, tiempo presente.
Materiales y metodología

- se define la muestra;

- se define el diseño, el método de análisis y el tratamiento de la información.

Resultados

- se presenta la información en correspondencia con los objetivos planteados;

- se exponen en una forma lógica y detallada los resultados obtenidos;

- se especifican las pruebas aplicadas para analizar los resultados;

- se emplean los términos del área de conocimiento a la que pertenece el tema de estudio;

- se utilizan las tablas, cuadros, gráficos para dar mayor claridad a la explicación;

- se citan las tablas, figuras cuadros, gráficos;

- se citan las referencias bibliográficas;

- se emplea la tercera persona, de modo impersonal, tiempo pasado;

- no se repite lo expuesto en la metodología.

Discusión

- se examinan e interpretan los resultados obtenidos en la investigación;

- se discute la relación y las contradicciones fundamentales de los datos obtenidos,

- se evalúan y califican las implicaciones de los resultados con respecto a las hipótesis originales.

\section{Conclusión}

- se expone si el estudio ha dado respuesta al problema planteado en la introducción;

- se expone a contribución del artículo;

- se plantean las conclusiones a las que se ha llegado; - se expresan las implicaciones teórico-prácticas que se pueden inferir del estudio.

Tipología textual. Organiza la gran variedad de usos lingüísticos. La clasificación se basa en los criterios siguientes: función, estructura y determinadas marcas lingüísticas, los cuales se reflejan en las características del texto. La tipología textual se clasifica en textos narrativos, descriptivos, explicativos, argumentativos, entre otros. 
Indicador de evaluación de la tipología textual

- selecciona la tipología textual adecuada a la función, los géneros discursivos y los marcadores lingüísticos del texto escrito.

En el caso del texto científico se trata de una tipología textual de tipo argumentativo, pero que contiene elementos explicativos, descriptivos, fundamentalmente. El esquema básico del texto es hipótesis-justificación- conclusión.

Léxico. Comprende el conjunto de vocablos, términos, nomenclatura, símbolos que emplea el profesional para plasmar las ideas, citerior, opiniones, datos, planteamientos, información y conocimientos.

Indicadores de evaluación del léxico

-emplea el léxico de un área del conocimiento específica;

-elije correctamente la terminología;

- utiliza cuando es imprescindible los sinónimos;

- evita el uso de palabras rebuscadas, ambiguas, anglicismos y barbarismos;

- evita el uso de palabras vagas, comodines, palabras en sentido figurado, superlativos;

- usa palabras concretas y evita las abstractas;

- minimiza el empleo de adverbios en -mente;

- limita el empleo de los gerundios;

- reitera el uso de palabras claves para reforzar conceptos y lograr precisión;

- evita el uso excesivo de abreviaturas, símbolos, fórmulas.

Morfosintáxis. La morfología y la sintaxis se necesitan recíprocamente. La primera atiende la formación, estructura y connotación de la palabra y la segunda se relaciona con elementos y construcción de la oración.

Indicadores de evaluación de la morfosintáxis

- marca de concordancia entre el artículo y el sustantivo, entre la persona y el verbo, entre el sujeto y el predicado $y$, en general, entre todos los componentes de la oración;

- elimina palabras innecesarias o redundantes en la oración;

• evita la cacofonía;
- construye las oraciones con los elementos imprescindibles;

- evita el uso abusivo del verbo ser y estar.

- ordena los elementos de la oración: sujeto, verbos y complementos;

-emplea oraciones cortas, breves y sencillas;

-usa la voz activa porque da claridad y concreción.

Ortografía. Parte de la gramática que enseña a escribir correctamente por el acertado empleo de las letras y de los signos auxiliares de la escritura.

Indicadores de evaluación de la ortografía

- diferencia la i-y, usa la m antes de b y p en los casos correspondientes;

- diferencia la b-v, la j-g, q-c-k, s-c-z-x, II-y;

-emplea correctamente la h;

- separa adecuadamente las sílabas;

- utiliza correctamente en los casos correspondientes las minúsculas y mayúsculas;

- marca la tilde regular en los casos correspondientes;

- diferencia las palabras parónimas.

Edición del texto. Es la organización de la apariencia estética. Se trabaja aspectos básicamente formales como tamaño de letra, colores, diagramas, gráficos, que pertenecen al diseño del documento para conseguir mayor claridad, legibilidad, coherencia y cohesión del texto.

Indicadores de evaluación de la edición del texto

- es adecuado a las restricciones del género;

- es acorde con las normas de la institución;

- favorece la claridad y la legibilidad del texto mediante tipos de letras, márgenes, interlineados, sangrías, justificaciones, disposición del texto, pertinencia de mayúsculas y minúsculas, contraste, uso de colores;

- es adecuado el uso de la tipografía para destacar elementos internos del texto: cursivas, negritas, subrayados, Versalitas, etc. para funciones específicas; tamaño de párrafos, sangrías, títulos, subtítulos, etc; - evita el uso de mayúsculas como elemento tipográfico; 


\section{Artículo de investigación}

- usa recursos como recuadros, viñetas para facilitar la legibilidad;

-emplea elementos gráficos para favorecer la claridad del texto; estos están numerados, se relacionan con el texto, están cerca de su mención en el texto, están acompañados de una leyenda, son comprensibles, interpretables y aportan información nueva, necesaria y relevante;

- es adecuado el uso de la numeración en la estructuración de las partes del texto: sistema numérico o arábigo y el alfanumérico;

-atiende la coherencia de las partes del texto;

- adopta una norma establecida para la edición de citas y referencias bibliográficas.

\section{Conclusiones}

La evaluación, desde la función formativa, permite recoger evidencias sobre el desarrollo del proceso de producción del texto científico y la calidad de este con vista a mejorar la comunicación científica escrita en el contexto universitario.

Para ello se proponen indicadores de evaluación relacionados con las fases del proceso de producción del escrito (planificación, textualización y revisión) y con el texto como producto final (contenido, habilidades cognitivas y cognitivo-lingüísticas, coherencia y cohesión, adecuación, género textual, tipología textual, léxico, morfosintáxis, ortografía y edición del texto). Los indicadores propuestos permiten al profesor y a los estudiantes valorar el proceso de producción del texto científico escrito y la calidad del mismo, además pueden servir de pauta para orientar el proceso de formación del profesional en comunicación científica escrita.

\section{Reconocimiento}

Proyecto «Estrategia didáctica para potenciar en los profesionales las habilidades comunicativas para la comunicación científica», Línea de investigación «Competencia comunicativa e intercultural en el estudio de lenguas». Facultad de Humanidades, Universidad Central «Marta Abreu » de Las Villas, Cuba.

\section{Bibliografía}

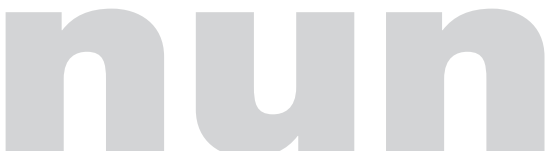

Albarrán, M. (2009) "Los indicadores de evaluación y los niveles de calidad de la composición escrita. Didáctica". En Lengua y Literatura, vol. 21 19-32 ISSN: 1130-0531.

Álvarez T. (2005) Didáctica del texto en la formación del profesorado. Madrid: Síntesis

Cassany, D. (1999) Construir la escritura. Barcelona: Paidos

Cisneros, M. (2003) Fundamentos de Redacción Técnica.Bogotá D.C: Unión Latina.

Flower, L. y Hayes, J. (1981) A Cognitive Process Theory of Writing. College Composition and Communication, 32, 365-387.

Martínez, C. (1994) Instrumentos de análisis del discurso escrito. Editorial Facultad de Humanidades. Colección Lengua y Cultura. Cali: Universidad del Valle.

Monné, P. (1998) "La escritura y su aprendizaje". En Mendoza, A. Conceptos clave en la didáctica de la lengua y literatura. Barcelona: Horsori. pp. 155-168

Morales, O. (2009) Los géneros escritos de la odontología Hispanoamericana. Barcelona. España. Tesis doctoral de la Universidad Pompeu de Fabra.

Parra, M. (s/f) Cómo se produce el texto escrito. Teoría y práctica. Bogotá D.C: Cooperativa Editorial Magisterio.

Roméu, A. (2002) "La comunicación en la ciencia". En Revista: Educación. Julio- diciembre. Ciudad de La Habana: Pueblo y Educación.

Roméu, A. (2008) La competencia investigativa y el dominio del discurso científico como objeto complejo en el posgrado. Universidad 2008. Cuba: La Habana

Serafini, M. (1999). ¿Cómo seescribe?.Barcelona:Paidós.

Tapia, M. y col. (2003) "Aplicación de una pauta diseñada para evaluar informes académicos universitarios". En Revista Signos, 36(54), 249-257

Teberosky, A. (2007). “El texto académico”. En Castelló, M. (Coord.) Escribir y comunicarse en contextos científicos y académicos. España: Graó.

Van Dijk, T. (1997) La ciencia del texto.Barcelona: Paidós. 